\title{
Nuevos modelos organizativos en torno a la biblioteca pública y las bibliotecas de prisiones
}

\author{
Margarita Pérez Pulido \\ José Luis Herrera Morillas \\ Universidad de Extremadura (España)
}

\section{Resumen}

Se realiza un estado de la cuestión sobre la situación actual de los servicios bibliotecarios en las prisiones. Se parte de un análisis de los documentos programáticos de la Unesco y la IFLA. Seguidamente, se estudian los modelos organizativos de Estados Unidos, Reino Unido y España, examinando las vinculaciones y dependencias, en cuanto a la organización de servicios, entre las instituciones penitenciarias, las educativas y las responsables del sistema bibliotecario. A partir de la información obtenida, se intenta definir un modelo teórico organizativo nuevo para España, que sirva para regularizar las bases de los acuerdos establecidos entre las bibliotecas públicas y las instituciones penitenciarias. Estos acuerdos se caracterizan por dejar bien sentados aspectos fundamentales de la organización de la biblioteca como centro de información, educación y ocio del centro penitenciario, y, en consecuencia, de la planificación y evaluación de la misma.

Palabras clave: Servicios de información para grupos en situación de desventaja. Bibliotecas públicas. Bibliotecas especiales. Bibliotecas de prisiones.

\section{Abstract}

A review of the situation of the library services in prisons is offered. First, the relevant documents of IFLA and UNESCO are examined to establish the basic programmatic frame. Thereafter, the organizational models of the United States, United Kingdom and Spain are analyzed, establishing their differences and similarities in how prison, educational and public libraries in the same district are articulated and cooperate. From this information, a theoretical model for Spain is proposed, that can be used to regulate the agreements that public libraries and prisons establish to ensure these services. These agreements set up the basic organization of the prison's library as an information center, an education facility and a leisure space, and, therefore, determine its planning and evaluation.

Keywords: Information services for disadvantaged groups. Public libraries. Special libraries. Prison libraries.

Scire. $13: 1$ (en.-jun. 2007) 33-50. ISSN 1135-3716. 


\section{Introducción}

Existe tradicionalmente una vinculación entre la biblioteca pública y los servicios de información para grupos en desventaja, ya que el Manifiesto de la Unesco (1994) define la biblioteca pública como una institución que debe proporcionar libre acceso a la información y la cultura a todas las personas sin distinción, e identifica en concreto a aquellos usuarios que merecen una atención especial y tienen derecho a un servicio de lectura pública. El fundamento de esta relación podemos encontrarlo en tres puntos que aparecen contemplados en dicho Manifiesto y son la finalidad de la biblioteca, la atención a diversa tipología de usuarios y la organización de su funcionamiento. Si establecemos una comparación entre las funciones, los usuarios y las normas de funcionamiento de la biblioteca pública y la biblioteca de prisión, observamos que, aunque partimos de un tronco común, los servicios de información para grupos en desventaja se caracterizan por poseer unas funciones más específicas dentro de las enunciadas para la biblioteca pública, unas condiciones particulares de la comunidad a la que sirven y una normativa de funcionamiento de acuerdo a estas circunstancias. Todo ello nos lleva a la necesidad de adoptar un modelo organizativo adecuado que haga efectivo el servicio ofrecido en función de estas consideraciones.

En este texto nos proponemos demostrar la especificidad de los servicios bibliotecarios de prisiones a partir del análisis de estos tres puntos iniciales, a la vez que realizamos un estudio de los diferentes modelos organizativos existentes al objeto de proponer el más adecuado a las circunstancias que se presentan en nuestro país.

\section{Biblioteca pública y servicios bibliotecarios de prisiones}

Las funciones de la biblioteca pública, de acuerdo con lo expresado en el Manifiesto de la Unesco, aparecen descritas en las Directrices IFLA/Unesco para el desarrollo del servicio de bibliotecas públicas (1) y, de forma sintetizada, son la educación e instrucción, la información, el perfeccionamiento personal, el progreso cultural (identidad cultural), la función social o la idea de la biblioteca como lugar de reunión e intercambio de ideas, y la responsabilidad con niños y jóvenes.

Por su parte, Lehmann (1999) concreta las funciones de una biblioteca de prisión en las siguientes (2): $a$ ) centro de lectura popular, entendiendo por este la provisión de materiales de lectura para el ocio; $b$ ) centro de aprendizaje independiente, en donde se ofrece la posibilidad de formación continua, información para aprender una profesión, servicios de referencia y ayuda para los cursos por correspondencia; $c$ ) centro de apoyo a la educación reglada, en los diferentes niveles y especialidades; $d$ ) centro de actividades de ocio y recreativas, con la posibilidad de organizar conferencias, tertulias, proyección de películas, exposiciones...; e) centro de información legal sobre cuestiones penitenciarias en general o de asesoramiento sobre casos específicos; $f$ ) centro de apoyo a los programas de rehabilitación, en

Scire. $13: 1$ (en.-jun. 2007) 33-50. ISSN 1135-3716. 
especial sobre drogodependencias y enfermedades contagiosas; $g$ ) centro de información sobre la comunidad exterior, con vistas a la reincorporación de los internos a la sociedad; $h$ ) centro de retiro personal donde se busque silencio e independencia; $i$ ) centro de apoyo al estudio, mediante la provisión de libros de texto $\mathrm{u}$ otros materiales; $j$ ) centro de investigación del personal, como centro de documentación sobre materias relacionadas con el trabajo.

Los servicios bibliotecarios de prisiones cumplen una misión de rehabilitación y reeducación de los internos, de modo que sus funciones adquieren un mayor grado de adaptación, al atender a un tipo de comunidad concreta dentro de las enumeradas para la biblioteca pública.

De igual modo, las Directrices IFLA/Unesco mencionadas cumplen lo expresado en el Manifiesto de la Unesco en cuanto a la necesidad de atender a todo tipo de usuarios, en la contemplación de la organización de servicios para grupos de usuarios especiales, que divide en aquellos que están recluidos en instituciones como prisiones u hospitales, personas con discapacidad o dificultades para aprender e inmigrantes, para conocer la nueva cultura o posibilitar el acceso a la suya propia. En las bibliotecas de prisiones, dentro de la comunidad reclusa, se encuentra representada toda esta tipología de usuarios considerados como especiales, además de población joven y femenina.

\section{Normas de servicios bibliotecarios de prisiones}

La especificidad de los servicios bibliotecarios de prisiones la encontramos de nuevo en su organización, ya que estos poseen normas de funcionamiento que concretan lo expuesto de un modo general en las Directrices IFLA/Unesco para el desarrollo del servicio de bibliotecas públicas.

El punto de partida de la elaboración de las normas son las disposiciones de carácter nacional e internacional que regulan los servicios bibliotecarios de prisiones (3). Todas coinciden en la necesidad de crear una biblioteca en cada centro penitenciario para el uso de toda clase de reclusos, formar una colección adecuada a las necesidades educacionales y recreativas de los mismos y estimular su uso.

Así pues, los principios fundamentales sobre los que se basa la elaboración de esta normativa son $a$ ) el derecho de los reclusos a acceder a la información dentro y fuera de su entorno; $b$ ) la consideración de los servicios bibliotecarios como parte del programa sociocultural del centro penitenciario; $c$ ) el apoyo a las actividades educativas del centro, al desarrollo personal y cultural de los reclusos, a su formación para el empleo y a la ocupación de sus momentos de ocio y distracción; $d$ la creación de un modelo organizativo similar al de una biblioteca pública; $e$ ) la necesidad de evaluar periódicamente el servicio para comprobar si cumple con los objetivos propuestos.

Scire. $13: 1$ (en.-jun. 2007) 33-50. ISSN 1135-3716. 
Las tres normativas estudiadas-Library standards for adult correctional institutions (ALA), Guidelines for prison libraries (LA), Guidelines for library services to prisoners (IFLA) (4)— hacen referencia a varias áreas de actuación: personal, presupuesto y financiación, equipamiento e instalaciones, colección, servicios, y promoción y difusión de los mismos. Consideramos interesante realizar una exposición de los puntos fundamentales en cada uno de los apartados mencionados.

Los aspectos tratados en las normas en el área de personal se refieren al grado de cualificación y los diferentes niveles administrativos de los bibliotecarios, su responsabilidad en función de ello, su número dependiendo de la cantidad de población atendida y las horas de dedicación. Se hace mención, igualmente, a las características y aptitudes que deben poseer, teniendo en cuenta el medio en el que han de trabajar y la necesidad, establecida por la ley penitenciara, de contar con reclusos como voluntarios.

Mientras que las pautas de la IFLA se limitan a decir que en un centro penitenciario la cantidad de personal variará en función de la población, habiendo al menos un bibliotecario responsable y cuantos se necesiten para organizar un buen servicio, contando en la medida de lo necesario con los internos voluntarios, las normas ALA y LA especifican todos los rangos en función de la población.

De este modo, la ALA contempla un bibliotecario profesional (titulado superior en Biblioteconomía y Documentación) por centro penitenciario, el cual debe realizar todas las tareas de coordinación y supervisión de servicios, y uno o dos ayudantes (diplomados o con conocimientos específicos) en función del número de internos del centro. En todos debe haber un auxiliar y de uno a tres administrativos.

En las normas de la LA, el personal se calcula en función de las horas semanales de dedicación. El tiempo de trabajo en los diferentes niveles dependerá de las horas de apertura de la biblioteca, la población del centro penitenciario, el nivel de uso de la biblioteca, la disponibilidad del bibliotecario profesional y las condiciones de seguridad del centro. Debemos tener en cuenta que los bibliotecarios son empleados de la biblioteca pública y dividen su jornada entre ambos centros. Para las instituciones penitenciarias que operan en el sector privado y contratan profesionales que no dependen de la biblioteca pública, la LA recomienda calcular las horas de dedicación en función de la tabla propuesta. En este caso, los directores de las prisiones deciden el nivel y el tipo de personal para su centro en función de la seguridad, el orden y la organización y funcionamiento de la biblioteca. Consideran fundamental que el profesional conozca y se familiarice con el entorno y establezca relaciones con otros departamentos de la prisión.

La LA incluye un programa específico de formación para cada puesto de trabajo y exige que se reconozca al responsable de la biblioteca como un miembro más 
del equipo de gestión del centro (5). Todas las normas coinciden en la necesidad de contar con suficiente personal en función de la población del centro (6). Este personal ha de estar debidamente cualificado, por lo que es necesario contar con programas de instrucción para todos los niveles.

La provisión de personal, según las normas de la IFLA, dependerá de la política penitenciaria de cada país; según las normas de la LA, ha de estar compartido con la biblioteca pública; y según las normas de la ALA, la responsabilidad de su contratación y supervisión recae en la biblioteca pública.

En cuanto al presupuesto y la financiación, las pautas de la IFLA otorgan al jefe de la biblioteca la misma autoridad que tienen las otras unidades de la institución para planificar y administrar dicho presupuesto. Hablan de una asignación de recursos permanente y suficiente para alcanzar las necesidades futuras del servicio. Establecen unos mínimos de compra de materiales y suscripciones a revistas y periódicos. La ALA designa para el servicio de biblioteca el $2 \%$ del presupuesto global del centro.

En el ámbito anglosajón, las normas de la LA se refieren a un presupuesto gestionado entre dos partes, la biblioteca pública y el centro penitenciario, a partir de los acuerdos establecidos en función de la población del centro (7). Como parte de este presupuesto compartido se encuentra lo relativo a la adquisición y provisión de servicios, el personal, el equipamiento, la colección para minorías étnicas y los procesos técnicos, además de partidas destinadas a la implantación de nuevas áreas de servicio.

Respecto a equipamiento e instalaciones, las normas recomiendan la superficie mínima que debe ocupar el espacio de la biblioteca y el número mínimo de puestos, en función de los internos en cada centro. Las pautas de la IFLA especifican que el espacio de la biblioteca debe ser suficiente para ubicar la colección y que hay que crear un área de lectura y trabajo e instalar exhibidores y otros medios de comunicación, así como un despacho para el bibliotecario. Se ha de contar con una serie de condiciones ambientales como iluminación, ventilación, tranquilidad y seguridad. Además la biblioteca debe estar dotada de instrumentos que faciliten el uso de materiales audiovisuales, así como el acceso a los catálogos manuales o automatizados.

De acuerdo con las normas de la LA, la biblioteca pública ha de ayudar a planificar el espacio de la biblioteca penitenciaria. Considera un mínimo de superficie de 93 metros cuadrados, además de establecer distancias mínimas entre los puestos de lectura. A igual que la IFLA, establece una distribución de espacios en función del fondo y los servicios proporcionados. Las características de ubicación de la biblioteca se basan en la flexibilidad para el cambio, la localización cerca del Departamento de Educación, los accesos externos y la decoración y dotación Scire. $13: 1$ (en.-jun. 2007) 33-50. ISSN 1135-3716. 
interna para crear una atmósfera adecuada. Las condiciones ambientales se refieren a la luz, el sonido y la ventilación (8).

Todas las normas coinciden en que el local debe cumplir unas condiciones mínimas de habitabilidad, muy bien especificadas en todas ellas, y contar con una diferenciación de espacios (distintos materiales para la sala de lectura, el despacho del bibliotecario...). Existen otras condiciones físicas especiales consideradas igualmente de manera general: acceso para discapacitados, control visual de toda la biblioteca y línea de teléfono propia.

Los procesos técnicos incluyen la catalogación, clasificación y mantenimiento de autoridades, el procesamiento de los materiales para la circulación y las técnicas para la promoción de los mismos. Se recomienda el uso de bases de datos electrónicas.

Las consideraciones de las tres normativas respecto a la colección giran en torno a tres cuestiones fundamentales: criterios de selección, tipos documentales y volumen de fondo.

En cuanto a la primera cuestión, todas las normas contemplan la necesidad de que la selección de fondos se realice de acuerdo con el perfil de la población reclusa, la política de actuación del centro penitenciario y las actividades que se organicen. Por otra parte, las normas de la LA especifican que la selección ha de hacerse en función del número de internos, el tiempo de permanenecia de estos en el centro y la posibilidad de acceso a la biblioteca. Las normas de la ALA basan la selección en las solicitudes de los internos, las consultas y el expurgo realizado. Se tienen en cuenta las necesidades específicas relacionadas con el mundo exterior. Las normas de la IFLA toman en consideración las demandas y solicitudes de los internos y del personal, así como los tipos de materiales que pueden servir de soporte a las actividades educativas del centro. Todas las normas tratan el tema de la censura: según la LA y la IFLA, solamente se aplica por motivos de seguridad, y según la ALA, en función de un uso excesivo de los servicios bibliotecarios.

Sobre los tipos documentales, las normativas están de acuerdo en incorporar toda clase de materiales, en diferentes soportes. La IFLA identifica 22 tipos distintos de materiales que deben encontrarse en una biblioteca de prisión.

En cuanto al volumen de fondo, todas las normas establecen un mínimo, en general y por tipo documental. Las normas de la IFLA son las que contemplan un mayor volumen de fondo. La normativa de la LA es mucho más precisa a este respecto, ya que especifica porcentajes para la renovación de fondo ( $15 \%$ anual, y en centros pequeños, $25 \%$ ) y el incremento anual de fondo (25\% en caso de tener bibliotecas de módulo), y considera las donaciones y el préstamo interbibliotecario como un complemento de la colección.

Para la difusión del fondo se recomienda el uso de catálogos y sistemas automatizados. Las normas de la LA consideran necesario tener, como mínimo, una

Scire. $13: 1$ (en.-jun. 2007) 33-50. ISSN 1135-3716. 
lista con suficiente información bibliográfica y diferentes puntos de acceso (autor, título, materia).

Las pautas de la IFLA contienen una sección específica para exponer sus consideraciones acerca de los servicios. En principio, en ella se indica que estos servicios deben estar en relación directa con los propósitos del centro penitenciario, de acuerdo con las necesidades individuales de la población reclusa. Se contemplan servicios especiales para aquellas personas que tengan alguna discapacidad física o mental y se establece la necesidad de acceso a lo disponible para quienes se encuentren en régimen de segregación, con base en una política escrita de servicio a fin de asegurar su cumplimiento. El programa establecido ha de ser amplio, y la comunicación con los usuarios, regular.

Igualmente se expone que se debe estudiar periódicamente las necesidades de los usuarios y establecer un programa de evaluación a fin de conocer la eficacia y eficiencia de los servicios. Las normas de la ALA conceden gran importancia a esta cuestión, y especifican que la evaluación será anual y los resultados habrán de ser presentados ante la Dirección del centro. Hablan de la necesidad de realizar estudios de usuarios para determinar la política del servicio en función de sus intereses.

Para evitar el aislamiento profesional, tanto la ALA como la IFLA recomiendan participar en redes y potenciar el préstamo interbibliotecario.

Las normas de la LA hacen hincapié en la organización adecuada de un servicio de referencia para los reclusos (9). Conceden una gran importancia al mantenimiento de un nivel de calidad en los servicios apropiado, para lo cual establecen la necesidad de incorporar indicadores de rendimiento, al igual que se hace para evaluar las acciones formativas.

En cuanto a los servicios recomendados, todas las normas coinciden en ofrecer aquellos que sirvan de soporte a las actividades realizadas en el centro penitenciario. Enfatizan la existencia de un servicio de información y referencia y de servicios especiales para minorías étnicas, culturales y segregadas, así como el establecimiento de redes de cooperación.

Otro aspecto fundamental que hay que analizar en relación con los servicios lo constituye el acceso a la biblioteca y a la colección, cuestión que preocupa especialmente a la LA.

Sobre el horario de apertura de la biblioteca y el tipo de visita, estas normas establecen un número de días y horas a la semana para que los internos puedan acceder a la biblioteca, en función de unos criterios: $a$ ) población, $b$ ) superficie de la biblioteca, $c$ ) tiempo de estancia en cada visita (20 minutos), $d$ ) número de visitas permitidas a la semana, $e$ ) uso de la sala de estudio y lectura de prensa, y $f$ ) horas de dedicación del bibliotecario profesional.

Scire. $13: 1$ (en.-jun. 2007) 33-50. ISSN 1135-3716. 
Las normas de la ALA limitan esta cuestión a las horas de dedicación del bibliotecario y las de IFLA no especifican nada.

Todas las normativas coinciden en ofrecer otras posibilidades de acceso a la colección, como son los carros, la distribución de listas de materiales o la creación de bibliotecas de módulo. Las pautas de la IFLA hablan de la necesidad de acercarse a los usuarios para que conozcan y utilicen los servicios bibliotecarios. Para ello se ha de crear una serie de instrumentos como bibliografías, recortes de prensa, listas de adquisiciones, folletos desplegables o información en otros idiomas. Igualmente se han de organizar actividades como conferencias, exposiciones, reuniones, talleres o seminarios. Las normas ALA comparten esta posición e insisten en la importancia que se debe conceder a la publicidad.

Para la LA, una de las cuestiones más importantes en cuanto a promoción se refiere a la señalización. Por ello especifica su organización en los diferentes niveles y da pautas acerca del tamaño, el estilo y el color de los signos, y de los mensajes contenidos en ellos. Obligatoriamente los reclusos han de disponer de una guía con información general de la biblioteca. Considera de gran importancia la formación de usuarios, basada sobre todo en sesiones de información básica sobre la biblioteca. Finalmente, hace referencia expresa a la necesidad de colaborar con el Departamento de Educación del centro.

\section{Modelos organizativos}

Una vez analizados los tres puntos sobre los que hemos fundamentado la relación entre biblioteca pública y biblioteca de prisión, pasamos a estudiar los diferentes modelos organizativos que hacen efectiva esta relación.

Los modelos organizativos de los diferentes países estudiados, que analizaremos con detenimiento a continuación, establecen dependencias entre las instituciones penitenciarias y las responsables del sistema bibliotecario a partir de convenios de colaboración entre los respectivos organismos administrativos que asumen competencias en estas materias.

\subsection{Modelo norteamericano}

Aunque los profesionales han intentado a lo largo de los años institucionalizar un modelo organizativo común (Coyle, 1987, p. 89), resulta imposible dada la estructura administrativa del país. De este modo, existen diferentes estructuras organizativas dependiendo de los estados y los sistemas federales de prisiones.

En algunos estados, por acuerdo entre instituciones culturales (State Library Agency) y penitenciarias (State Correctional Agency), la biblioteca de prisión se constituye como una sección de la biblioteca pública. El objetivo fundamental es el de proporcionar servicios bibliotecarios de calidad para satisfacer las necesidades informativas, educativas y de ocio de los reclusos. Desde este punto de vista, la bi- 
blioteca pública contrata y supervisa el personal bibliotecario; planifica, supervisa y coordina el servicio bibliotecario ofrecido al centro; y ofrece algún presupuesto para la adquisición de materiales. Por su parte, el centro penitenciario se compromete a dotar la biblioteca de espacio y equipamiento suficiente, crear una biblioteca legal —requisito obligatorio en el sistema penitenciario americano- y establecer los procedimientos adecuados de conocimiento y trabajo en un entorno penitenciario. Todo ello se realiza mediante la conexión y coordinación entre el Institutional Library Service y el Correctional Programming, de cuya unidad depende la biblioteca del centro penitenciario.

La biblioteca pública ofrece tres posibilidades: $a$ ) crear una sección en el centro penitenciario en donde se ofrezcan todos los servicios bibliotecarios, con materiales catalogados y puestos a disposición en una base de datos, atendida por un profesional de la biblioteca pública durante 20-40 horas a la semana; $b$ ) lo que denominan library station, con servicios parciales, materiales no catalogados ni ofrecidos en base de datos y sostenida por reclusos con supervisión de un profesional bibliotecario; c) lotes de libros no catalogados colocados en una sección de la prisión.

Las categorías de personal contempladas en estos servicios son igualmente variadas: por un lado están los bibliotecarios especializados contratados por la biblioteca pública (public library staff members); por otro, los bibliotecarios responsables que proporcionan libros y servicios (librarian) y los bibliotecarios profesionales con titulación en biblioteconomía y documentación (professional librarian); y, finalmente, los voluntarios y los propios reclusos del centro.

El segundo modelo aplicado supone, igualmente, la colaboración con la biblioteca pública. En este caso, el presupuesto se divide entre las dos instituciones (State Library Agency y State Correctional Agency) y la biblioteca pública aporta asesoramiento y servicio a partir de la conexión entre el Institutional Library Service y el Correctional Programming. Este compromiso supone establecer acuerdos cerrados sobre el funcionamiento de la biblioteca, lo cual, generalmente, ha dado lugar a conflictos entre las partes, por lo que en realidad son pocos los estados que mantienen esta fórmula.

El modelo más comúnmente aceptado por el sistema norteamericano de prisiones es el que presenta un funcionamiento independiente del servicio bibliotecario del centro, considerando la biblioteca pública como un órgano de consulta por parte de los profesionales que están al cargo del servicio. La implantación de este modelo depende generalmente de la Dirección del centro y ha de contar con bibliotecarios profesionales.

\subsection{Modelo anglosajón}

El modelo organizativo anglosajón (10) se basa igualmente en la colaboración de las instituciones penitenciarias con las que conforman el sistema de lectura pública (Johnston y Byrne, 1992).

Scire. $13: 1$ (en.-jun. 2007) 33-50. ISSN 1135-3716. 
La Public Libraries and Museums Act, de 1964, establece ya la obligatoriedad de proporcionar un servicio bibliotecario a todas las personas en cooperación con las instituciones competentes, por lo que las bibliotecas públicas asumen la necesidad de incorporar las bibliotecas de prisiones como una sección más que han de atender.

El Home Office, institución administrativa encargada del sistema de prisiones, publica en 1978 las Library Facilities for people in custody, donde se establece la obligación de crear una biblioteca en todos los centros penitenciarios. Esta ha de formar parte del Departamento de Educación (Education Department) y, aunque se considera independiente de la escuela de la prisión, constituye un fuerte soporte para los docentes del centro. El documento Developments in prison service education and training, publicado en 1990, especifica que la biblioteca ha de contribuir a la formación y educación de los reclusos en colaboración conjunta con la escuela.

Otra normativa aparece como consecuencia de las anteriores: es la elaborada en 1981 por la Library Association, Prison libraries: guidelines for library provision in prison department establishments, que establece la colaboración de ambas instituciones en la organización de los servicios y la adaptación a las condiciones especiales de la prisión.

Así pues, la biblioteca pública local es la encargada de organizar el servicio bibliotecario del centro penitenciario más próximo, de modo que se establecen las relaciones oportunas entre el Home Office Prison Service y el County Library Service.

El Home Office Prison Service concede una asignación presupuestaria anual a la biblioteca pública basada en una fórmula creada para calcular el coste del servicio (materiales, mantenimiento, procesos y servicios, formación). Esta asignación aumenta anualmente en proporción al número de reclusos del centro penitenciario. Igualmente, proporciona el salario de un bibliotecario a tiempo parcial (de 8 a 12 horas por semana). Este será un bibliotecario titulado sujeto a las condiciones de servicio de la biblioteca pública pero dependiente, dentro del centro penitenciario, del funcionario de Educación, según lo reglamentado por la Dirección del centro.

La County Library coordina en general todo el servicio y ofrece a la biblioteca un servicio libre de referencia para los reclusos, el catálogo completo de la biblioteca pública en microficha actualizado semanalmente, junto a un ejemplar en microficha de los British books in print actualizados mensualmente, intercambio de fondos con la biblioteca pública, catalogación de donaciones, posibilidad de acceso a todos los servicios de la biblioteca pública, participación en actividades de difusión y formación de las personas encargadas de la biblioteca.

El personal de la biblioteca interviene en diferentes niveles. El assistant county librarian establece la política del servicio de la biblioteca. El assistant area librarian se responsabiliza de las operaciones de provisión y coordinación del ser- 
vicio. Los prison librarians se ocupan de la selección y adquisición, la formación de los reclusos y el desarrollo y promoción de los servicios. Forman parte del personal de la biblioteca pública, y por eso están bajo la supervisión del assistant area librarian, pero, dentro de la prisión, dependen orgánicamente del education officer. De hecho, su salario procede del presupuesto de educación, contabilizado como horas de enseñanza.

El personal del centro penitenciario implicado en la organización del servicio bibliotecario los constituyen el prison educator officer, que se encarga de la vigilancia en general, de representar los intereses del centro y de asegurar la tranquilidad del bibliotecario en su trabajo; el library officer, que mantiene la disciplina entre los reclusos y el buen uso de la biblioteca; y los inmates orderlies, encargados de los trabajos rutinarios, la comunicación entre bibliotecarios y reclusos y los procesos técnicos diarios como el préstamo o la colocación del fondo.

Una vez al mes se reúne el Library Management Committee, órgano consultivo y de toma de decisiones a través del cual se ponen en contacto ambas instituciones.

Según un informe realizado sobre la situación de los servicios bibliotecarios de prisión en Leicestershire (1989), la aplicación de este modelo organizativo supone revisar algunas cuestiones, tales como la mayor necesidad de contacto entre bibliotecarios y reclusos, una mayor formación de los encargados de las bibliotecas y los reclusos ayudantes, la limitación en el compromiso de la biblioteca con el Departamento de Educación y una gestión más coordinada entre las instituciones implicadas.

En 1994 se produce una reestructuración en la organización de los servicios penitenciarios con la finalidad de mejorar la coordinación entre las autoridades responsables de la educación en prisión. De este modo, la Chief Education Officer's Branch (CEOB) se reorganiza en el Education and Training Advisory Service (ETA) junto con los Prison Enterprise and Activity Services (PEAS). El principal education officer será a partir de ahora el chief education and training adviser, y el principal college assistant será el education and training adviser. Un miembro del grupo continúa con la responsabilidad de las bibliotecas de prisiones.

\subsection{Modelo español}

El modelo organizativo español es similar al de otros países mediterráneos como Francia (11) o Italia (Manganelli, 1989), en los que las bibliotecas de prisiones dependen en cuanto a gestión y funcionamiento de los instituciones penitenciarias, y se establecen acuerdos de cooperación de carácter general con instituciones y organismos públicos y privados. La colaboración con la biblioteca pública más cercana al centro penitenciario no se realiza de forma generalizada, sino en algunos casos concretos (12), debido a la voluntad de las personas al frente de la dirección de estas bibliotecas o del propio centro penitenciario.

Scire. $13: 1$ (en.-jun. 2007) 33-50. ISSN 1135-3716. 
El Acuerdo de Cooperación Bibliotecaria firmado en diciembre de 1983 entre ambos ministerios (Justicia y Cultura) supone la dotación de las bibliotecas de todos los centros penitenciarios españoles, así como un compromiso de cooperación en la gestión por parte de las bibliotecas que conforman el sistema bibliotecario del Estado.

Por este acuerdo, se establece que el Ministerio de Cultura habrá de colaborar con los centros penitenciarios comprometiéndose a asesorar a los encargados de las bibliotecas y recibir estadísticas trimestrales, además de incrementar periódicamente los fondos bibliográficos. Por su parte, el Ministerio de Justicia se compromete a dotar de infraestructura y mobiliario a todas las bibliotecas dependientes, poner al frente a un profesor de EGB, organizarlas según las normas técnicas biblioteconómicas, fomentar su uso entre los internos y facilitar las visitas de los directores de las bibliotecas públicas.

A partir de ese momento y hasta la actualidad, se ha ido renovando los acuerdos establecidos inicialmente, preparando nuevos lotes de libros, realizando estudios sobre la situación de las bibliotecas de prisiones y organizando cursos de formación bibliotecaria.

Por su parte, la legislación penitenciaria (13) prevé la existencia de una biblioteca en cada centro, con salas de lectura suficientes atendiendo a su estructura modular, y contempla la posibilidad de utilizar libros facilitados por los servicios bibliotecarios ambulantes establecidos por la Administración o por entidades particulares. Dispone su dependencia orgánica de la unidad docente del centro y su vinculación a la escuela, sobre todo al considerar a un maestro como la persona encargada de su gestión, con la ayuda de internos auxiliares. Las normas de funcionamiento provienen de instrucciones procedentes del Organismo Autónomo de Trabajo y Prestaciones Penitenciarias, y tratan de cumplir lo que especifica la normativa penitenciaria en cuanto a fondos, procesos técnicos, adquisiciones y servicios (14). La biblioteca no cuenta con presupuesto propio, por lo que depende de las asignaciones de la Dirección General de Instituciones Penitenciarias por una parte y del propio centro por otra.

Sin embargo, a partir de 1999 se introduce una nueva reglamentación (15), por la cual la biblioteca pasa a depender directamente de la Subdirección de Tratamiento del centro penitenciario a través de una nueva figura creada denominada coordinador de formación, de modo que se desvincula orgánicamente de la unidad docente. Este cambio organizativo ha supuesto la contratación de bibliotecarios profesionales en 11 prisiones y la edición de unas normas generales de funcionamiento de la biblioteca, aunque no adaptadas a las analizadas anteriormente.

Por lo que respecta a la participación de los organismos competentes de las comunidades autónomas a partir del desarrollo de su propia legislación en materia de 
cultura, las normativas contemplan la asistencia a grupos en situación de desventaja, y algunas de ellas consideran las bibliotecas de prisiones como una tipología más de bibliotecas que debe incluirse dentro de su sistema bibliotecario.

De este modo, el Principado de Asturias contempla, dentro del plan de bibliotecas de su comunidad, el desarrollo de servicios bibliotecarios en establecimientos penitenciarios, entre otros, aunque concede prioridad a los servicios de carácter general (16). Castilla y León considera la promoción de programas de extensión bibliotecaria para centros penitenciarios a través de los centros integrados en el sistema bibliotecario comunitario (17). La Comunidad de Murcia recomienda el desarrollo de servicios bibliotecarios en establecimientos penitenciarios (18). Galicia realiza una declaración de intenciones al expresar, en el artículo 10 de la Ley de Bibliotecas, que "velará para que los servicios bibliotecarios, en su conjunto, puedan atender adecuadamente a grupos específicos de usuarios" (19).

En el caso de la Comunidad Autónoma de Cataluña, en el año 1983, por real decreto, se producen las transferencias del Estado a la Generalitat en materia de administración penitenciaria, lo que se traduce en una total libertad de acción para la gestión de las bibliotecas por parte del Gobierno autónomo y de cada centro penitenciario (20). Las bibliotecas dependen orgánicamente de la Subdirección de Tratamiento del centro penitenciario y forman parte del denominado Programa de Educación No Formal dentro del área de educación; están exentas de la dependencia de la escuela pero colaboran estrechamente con ella como parte del Programa Específico de Bibliotecas. Todos estos programas son elaborados y supervisados por la Sección de Educación, Cultura y Deporte, perteneciente al Servicio de Rehabilitación, en la Dirección General de Servicios Penitenciarios, dentro del Departamento de Justicia de la Generalitat (21).

Todas las prisiones de Cataluña cuentan en la actualidad con un bibliotecario profesional, diplomado en Biblioteconomía y Documentación, que tiene un salario fijo y un horario administrativo completo que ha de cumplir dentro del centro penitenciario (22). Las tareas de difusión las comparten con las unidades docentes, los procesos técnicos son revisados y se han dictado normas de actuación por parte del organismo del que dependen (Sección de Educación, Cultura y Deporte). El modelo organizativo catalán contempla igualmente la colaboración con agentes externos a fin de completar sus servicios.

La Ley 4/1993, de 18 de marzo, del Sistema Bibliotecario de Cataluña (23) supone una novedad en esta comunidad respecto a la ley anterior en cuanto a la ordenación territorial (comarcas y municipios) y la unificación en un solo sistema de lectura pública de las redes de bibliotecas existentes dependientes de las diferentes administraciones, al igual que en la consideración de la totalidad de los servicios especiales como responsabilidad de la biblioteca pública y el establecimiento de una cooperación con los servicios de asistencia social de la comunidad.

Scire. $13: 1$ (en.-jun. 2007) 33-50. ISSN 1135-3716. 


\section{Un nuevo modelo organizativo}

El análisis de la situación de los servicios bibliotecarios de prisiones en España (Pérez Pulido y Sulé Duesa, 2004) nos permite afirmar que el actual modelo organizativo no funciona correctamente. De esta situación se derivan varias problemas: personal no especializado al cargo de estas bibliotecas, ausencia de presupuesto propio, dotación irregular de fondos, formación igualmente intermitente, organización de los servicios en función del personal que se encuentra a su cargo, ausencia de normas técnicas efectivas y colaboración voluntaria de la biblioteca pública más cercana al centro penitenciario. En consecuencia, proponemos un nuevo modelo organizativo para nuestro país basado en convenios entre instituciones culturales y penitenciarias, tal y como se ha realizado hasta el momento, pero formalizados en este caso a través del sistema público de lectura de las diferentes comunidades autónomas.

Las instituciones penitenciarias se hacen cargo del mantenimiento de una persona responsable al frente de la biblioteca, la dotación de infraestructura y presupuesto, y la organización del servicio bibliotecario en cada centro penitenciario. Por su parte, la Comunidad Autónoma, a través de sus órganos competentes, ofrece el asesoramiento y la formación del personal, la actualización de la colección y la participación en redes bibliotecarias, además de la colaboración con la biblioteca pública más próxima al centro penitenciario.

Si trasladamos este modelo a un ejemplo concreto, en la Comunidad Autónoma de Extremadura, la Ley 6/97, de 29 de mayo, de Bibliotecas de Extremadura (24) permite la integración de una biblioteca en el sistema bibliotecario mediante la firma de un convenio. De este modo, una vez formalizado el convenio con las instituciones penitenciarias, las dos bibliotecas de prisiones existentes en esta comunidad autónoma, una en Cáceres y otra en Badajoz, pasarían a formar parte del sistema público de lectura de la comunidad y podrían acceder a servicios y ayudas de la Consejería de Cultura. El Decreto 134/1998 (25) establece el procedimiento de incorporación de centros y servicios al sistema bibliotecario de Extremadura, por lo que las instituciones penitenciarias habrían de tramitar la solicitud de integración según el procedimiento indicado y cumplir unos requisitos mínimos de colección y servicios, avalados por una certificación técnica de la biblioteca, además de comprometerse a elaborar unas normas de funcionamiento internas adaptadas a las comunidad, aprobadas por la Consejería de Cultura, y remitir periódicamente estadísticas a la misma. Por su parte, los centros penitenciarios aportarían personal, infraestructura, parte del presupuesto y organización del servicio.

Este decreto especifica, además, que aquellas bibliotecas integradas en los antiguos centros coordinadores de bibliotecas se incorporan formalmente al sistema actual mediante oficio, lo que sucede con la biblioteca del centro penitenciario de 
Cáceres, no así con la del de Badajoz, la cual resultaría beneficiada por las ventajas de este nuevo modelo organizativo.

\section{Conclusiones}

Existe una vinculación entre la biblioteca pública y los servicios bibliotecarios de prisiones, aunque reconocemos en estos últimos unas funciones, usuarios y normas de funcionamiento más específicos, que requieren una particular atención en cuanto a su gestión.

Las normas de funcionamiento de los servicios bibliotecarios de prisiones reflejan la necesidad de procurar una relación estrecha entre todas instituciones implicadas. De hecho, en las normativas estudiadas se aprecia la influencia de las estructuras organizativas del sistema penitenciario vigente y sus relaciones con otras instituciones marcadas por la ley, además de consideraciones sociales y culturales propias de cada país.

Los modelos organizativos analizados basan esta vinculación en la formalización de convenios entre las instituciones culturales y las penitenciarias que permiten un funcionamiento independiente o dependiente de la biblioteca de prisiones respecto a la biblioteca pública. Las relaciones entre instituciones en el modelo gestión se fundamentan en la aportación de servicios y asesoramiento por una parte y de recursos por otra, aunque existen casos donde se combinan ambas posibilidades.

En nuestro país, la solución para la adecuada gestión de estas bibliotecas viene dada por su integración de manera formal en los sistemas bibliotecarios de las comunidades autónomas, al objeto de que la colaboración de las instituciones culturales no quede en una mera declaración de intenciones y se regularicen los acuerdos de dotación y prestación de servicios. Las instituciones penitenciarias, y los centros penitenciarios en particular, deben asumir su parte de compromiso al formalizar estos convenios, en el convencimiento de que la cooperación resulta esencial, tal y como se plantean los servicios bibliotecarios en la actualidad.

\section{Notas}

(1) Para una mayor comprensión de estas funciones véanse las Directrices IFLA/Unesco para el desarrollo del servicio de bibliotecas públicas (2002, pp. 2-8).

(2) Este autor establece las funciones de una biblioteca de prisión a partir de un modelo de planificación de bibliotecas de prisiones desarrollado por Rubin (1997).

(3 Recordemos la Regla 40 de las Naciones Unidas para el tratamiento de los prisioneros, las disposiciones del Consejo de Europa y la legislación propia de cada uno de los países en materia penitenciaria, además de las consideraciones de las asociaciones profesionales de bibliotecas.

Scire. $13: 1$ (en.-jun. 2007) 33-50. ISSN 1135-3716. 
(4) Las normas americanas publicadas en 1981 fueron muy criticadas por los profesionales de las bibliotecas, que opinaban que muchas de las cuantificaciones realizadas, sobre todo en lo que respecta a la colección, superficie y presupuesto, sobrepasaban las posibilidades de los centros penitenciarios y, por tanto, no se ajustaban a la realidad. Por este motivo se reformaron y se publicaron de nuevo en 1992 (American Library Association, 1992; Collins y Boden, 1997; Kaiser, 1992). En 1993 se publicó una edición traducida al castellano, y en 1995 una segunda edición, cuya novedad consiste fundamentalmente en la ampliación de la bibliografía final y la introducción de un capítulo acerca de la misión y las funciones de este tipo de biblioteca (Kaiser, 1995). Está prevista una tercera edición.

(5) Los apéndices 6, 7, 8 y 9 de la segunda edición de las normas describen las funciones para cada uno de los niveles profesionales y la habilitación de un plan de formación.

(6) LeDonne (1977, p. 69) considera que el capital humano es esencial para el adecuado funcionamiento de un servicio, ya que aporta conocimientos específicos y ciertas cualidades humanas.

(7) En el apéndice 5 de la segunda edición de las normas se establecen los acuerdos de pago a la biblioteca pública para compensar la pérdida de materiales ofrecidos en préstamo.

(8) Apéndice 10 de la nueva edición de las normas.

(9) En el apéndice 2 de la segunda edición de las normas se redactan unas consideraciones acerca de la organización de un servicio de información a la comunidad. En el apéndice 11 se establece la relación de documentos legales que deben poseer obligatoriamente todas las bibliotecas.

(10) Entendemos por ámbito anglosajón, en este caso, Inglaterra y Gales. En Escocia y en Irlanda del Norte la responsabilidad de los centros penitenciarios recae respectivamente en la Scottish Office y en la Northern Ireland Office. Para obtener más información sobre este asunto consúltese Wallace (1988), Prison library service in Oxfordshire, 1989 (1990) y Prison library services in Devon (1985).

(11) En este país se establecen por primera vez acuerdos serios entre el Ministerio de Justicia y el de Cultura en 1986. A partir de las disposiciones comunitarias de 1989 se elaboran normativas como la Circular AP92-08 sobre el funcionamiento de las bibliotecas y el desarrollo de las prácticas de lectura en los establecimientos penitenciarios. Se crean convenios de colaboración entre los distintos ministerios y las colectividades locales, pero los términos de esta colaboración no se encuentran debidamente reglamentados.

(12) Véase el caso concreto de colaboración entre la prisión de Guadalajara y la biblioteca pública en el artículo de Blanca Calvo (1997).

(13) Art. 57 de la LGOP y arts. 127, 128 y 129 del RP.

(14) Arts. 176, 177 y 178 del RP y arts. 52.5 y 57 del mismo reglamento.

(15) Véase el Real Decreto 1203/1999, de 9 de julio, por el que se integra en el cuerpo de maestros a los funcionarios pertenecientes al cuerpo de profesores de educación general básica de instituciones penitenciarias y se disponen normas de funcionamiento de las unidades educativas de los establecimientos penitenciarios $(B O E, 173$, de 21 de julio de 1999), y las instrucciones del Organismo Autónomo Trabajo y Prestaciones Peni- 
tenciarias sobre el funcionamiento de este mismo organismo (I9/1999 TP) y sobre los criterios a seguir para elaborar la programación de actividades de los centros penitenciarios del curso 2001/2002 (I8/2001TP).

(16) Decreto 68/86, de 15 de mayo, por el que se establecen las normas generales de actuación del Principado de Asturias para la promoción y coordinación de servicios bibliotecarios (BOPA, 142, de 19 de junio de 1986).

(17) Ley 9/1989, de 30 de noviembre, de Bibliotecas de Castilla y León (BOCYL, 224, de 22 de diciembre de 1989).

(18) Ley 7/1990, de 11 de abril de Bibliotecas y Patrimonio Bibliográfico de la Región de Murcia (BOE, 171, de 18 de julio de 1990).

(19) Ley 14/1989, de 11 de octubre, de Bibliotecas (DOG, 204, de 24 de octubre de 1989), tít. III, art. 10.

(20) Véase el Real Decreto 3482/83, de 28 de diciembre, sobre traspaso de servicios del Estado a la Generalitat en materia de administración penitenciaria (BOE, 43, de 20 de febrero de 1984), y el Real Decreto 131/86, de 10 de enero, sobre ampliación de medios adscritos a los servicios de la Generalitat en materia de administración penitenciaria (BOE, 28, de 1 de febrero de 1986).

(21) Véase el Decreto 259/1990 de creación de la Subdirección General de Asuntos Penitenciarios como parte de la Dirección General de Servicios Penitenciarios y Rehabilitación (DOGC, 1365, de 9 de noviembre de 1990) y el Decreto 106/1995, de 7 de mayo, sobre reestructuración de la Dirección General de Servicios Penitenciarios y de Rehabilitación (DOGC, 2038, de 12 de abril, de 1995).

(22) En la actualidad, 9 de los 10 centros penitenciarios existentes en Cataluña cuentan con un bibliotecario titulado en Biblioteconomía y Documentación.

(23) Se publicó en el BOGC, 1727, de 29 de marzo de 1993, y supone la modificación y puesta al día de la anterior Ley 3/1981, de 22 de abril, de Bibliotecas (DOGC, 123, de 28 de abril de 1981).

(24) DOE, 75, de 28 de junio de 1997.

(25) DOE, 134, de 21 de noviembre de 1998.

\section{Referencias}

American Library Association (1992). Library standards for adult correccional institutions. Chicago: ALA, 1992.

Bars to books-prison libraries in Leicestershire: a review of services (1989). Leicestershire: Libraries and Information Service, 1989.

Calvo, B. (1997). Sendas de Libertad. // Educación y Biblioteca. 85 (1997) 57-60.

Collins, R.; Boden, L. (eds.) (1997). Guidelines for prison libraries. Londres: Library Association, 1997. 2. ${ }^{\mathrm{a}}$ ed.

Coyle, W. J. (1987). Libraries in prisons: a blending of institutions. Nueva York: Greenwood Press, 1987.

Directrices IFLA/Unesco para el desarrollo del servicio de bibliotecas públicas (2002). Madrid: Ministerio de Educación, Cultura y Deporte, 2002, pp. 2-8.

Scire. $13: 1$ (en.-jun. 2007) 33-50. ISSN 1135-3716. 
Johnston, A.; Byrne, J. (1992). Dorset: the inside story. Prison libraries in Dorset: a professional appraisal of prison library services carried out by Dorset County Library during 1991/2. Dorset: County Library, 1992.

Kaiser, F. (ed.) (1992). Guidelines for library services to prisoners. La Haya: IFLA, 1992.

Kaiser, F. (ed.) (1995). Guidelines for library services to prisoners. La Haya: IFLA, 1995. $2 .^{\mathrm{a}} \mathrm{ed}$.

LeDonne, M. (1977). Survey of library and information problems in correctional facilities: a retrospective review. // Library Trends. 26:1 (1977) 53-70.

Lehmann, V. (1999). Se necesitan bibliotecarios de prisiones: una profesión estimulante para personas con las destrezas personales y profesionales adecuadas. // $65^{\text {th }}$ IFLA Council and General Conference (Bangkok, August 20-28, 1999). http://www.ifla.org/IV/ ifla65/papers/046-132s.htm (1999-11-08).

Manganelli, V. (1989). Le biblioteche carcerarie. // Biblioteche Oggi. 7:6 (nov.-dec. 1989) 731-736.

Manifiesto de la UNESCO sobre la biblioteca pública 1994. Salamanca: Diputación; Fundación Germán Sánchez Ruipérez, 1995.

Pérez Pulido, M.; Sulé Duesa, A. (2004). Las bibliotecas de prisión en España: una propuesta de mejora de la gestión y el aprovechamiento de los recursos. // Foro Biblioteca y Sociedad: experiencias de innovación y mejora. Murcia: Universidad, 2004. http://www.anabad.org/admin/archivo/docdow.php?id=156.

Prison library service in Oxfordshire (1990). Oxfordshire: Library Service, 1990.

Prison library services in Devon: a review (1985). Devon: County Council, 1985.

Rubin, R. J. (1997). Planning process for Wisconsin institution libraries: a workbook. Oakland, CA: Rubin Consulting, 1997.

Wallace, M. (1988). Breaking out! Library service to penal institutions. // Scottish Libraries. 7 (Jan.-Feb. 1988) 3-5. 